\title{
Hydrogen Detection With FBG Sensor Technology for Disaster Prevention
}

\author{
Said $\mathrm{SAAD}^{1^{*}}$ and Lotfi HASSINE ${ }^{2}$ \\ ${ }^{1}$ Group of Electronics and Quantum Physics Laboratory of Advanced Materials and Quantum Phenomena, Faculty of \\ Sciences of Tunis, 2092, Tunisia \\ ${ }^{2}$ National Institute of Applied Sciences and Technology University of Carthage, North Urban Center of Tunis-B.P.676 \\ Cedex Tunis-1080, Tunisia \\ *Corresponding author: Said SAAD_Ｅ-mail: saad.said.bechir@gmail.com
}

\begin{abstract}
Three fiber Bragg grating (FBG) sensors systems for hydrogen detection are presented using the classic Pd (palladium) coating technique $(500 \mathrm{~nm})$ as the hydrogen sensitive film and titanium (Ti) as the adhesive layer with the etched cladding. These systems are compared and used for disaster environment prevention in hydrogen leakage environment where higher values than the normal are presented which increase the risk of explosion. With these systems, $0.1 \%-4 \%$ of the hydrogen volume concentration range in the volume ratio was detected and monitored experimentally in the test room with the very sensitive and stable value which reached $60 \mathrm{pm} / 1 \% \mathrm{H}_{2}$. In addition, a fast response time, about $6 \mathrm{~s}$, was obtained with an advanced sensor.
\end{abstract}

Keywords: Fiber Bragg gratin (FBG), hydrogen detection, sensor, disaster environment prevention, Pd coating technique

Citation: Said SAAD and Lotfi HASSINE, "Hydrogen Detection With FBG Sensor Technology for Disaster Prevention," Photonic Sensors, DOI: 10.1007/s13320-013-0109-4.

\section{Introduction}

In this time, the disaster environment increases in a huge way, like the earthquake and tsunami, due to a rapid change in the climate. The most recent disaster that is known by the mankind is absolutely the great earthquake and tsunami that hit Japan on March 11, 2011, which caused about 20,000 dead and missing people and also caused Japan's worst nuclear accident at Fukushima Daiichi [1]. Therefore, actually some countries : USA, Japan, the Republic of Indonesia, etc. and large research companies seek for creating the ultimate technology that really contributes to avoid the tragedy to happen again. The technology found that can adapt to such challenges is "the disaster prevention photonics", whose recent development in Japan will be introduced. The main goal of this technology is to develop and fuse the technologies of the highly sensitive optical sensors for environmental monitoring, including the climate change and diastrophism that might lead to disasters, photonic devices that can work even under adverse conditions such as radiation environment, and photonic network that really functions at the time of the disaster. The photonic component that can be used to solve all the challenges and the requirements mentioned before is absolutely the optical fiber.

Received: 18 January 2013 / Revised version: 23 February 2013

(C) The Author(s) 2013. This article is published with open access at Springerlink.com 
Especially, the recent technology of the fiber Bragg grating (FBG) is used as a sensor and as a structural health monitoring system (SHMS) due to its advantages like high sensitivity, high accuracy, intrinsic safety, multiplexing like wavelength division multiplexing (WDM), immunity to the electrical and magnetic interference, both optical communications with optical sensing.

In the last decade, the FBG technology had a large development in the technique and manufacturing process [2] to make the desired opportunity of the required applications possible and to make the response to the measurand be linear within a measured interval. Nevertheless, the FBGs-based sensor incurs the problem of the grating decay and instability with time, which is in particular grave, when FBGs work always under stress and at an elevated temperature. To overcome this difficulty, several researches on novel photosensitive fiber structures and grating fabricating techniques were expansively carried out in the past decade [3, 4]. In this paper, three types of FBGs will be used: conventional FBG, chirped FBG, and special chirped FBG. The last type was developed by our research group, which is suitable, from the obtained results, for both optical communications and optical sensing even under random fluctuations of their internal parameters during construction.

On the other hand, hydrogen is a clean energy and an important chemical raw material, which has been widely used in many industrial fields such as aerospace, fuel cells, metal smelting and chemical synthesis. However, hydrogen gas is so easy to leak because of its smallest molecule, which may explode when its concentration reaches to a certain value, $4 \%$ of the concentration, in the volume ratio in the closed environment. Therefore, it is extremely important to monitor the hydrogen leakage in these fields. In this paper, we use FBGs as sensors for monitoring and detecting this hydrogen leakage. Three sensors were developed, and each one used one type of FBGs mentioned with the etched cladding. These sensors could not be achieved without the palladium, $\mathrm{Pd}$, coating technique as the hydrogen sensitive film was deposited on the length of each FBG to enhance the hydrogen molecules detection, precisely increasing the absorption of hydrogen molecules. In addition, we found in [5] another coating solution which was Pt-loaded $\mathrm{WO}_{3}$ (tungsten trioxide) which showed also good results. In the same line, the first hydrogen FBG sensor was reported by Sutapun et al. [6]. However, our Pd layer was realized with magnetron sputtering to get the high sensitivity, and the shift in the fiber Bragg wavelength was observed for the three sensors with different gas hydrogen concentrations, $0.1 \%-4 \%$ volume concentration range, which was caused by the decreasing complex refractive index of Pd with increasing absorption of hydrogen. Experimental results showed that the chirped FBG hydrogen sensor demonstrated the highest sensitivity especially in the low gas hydrogen concentration where the sensitivity was raised up to $60 \mathrm{pm} / 1 \% \mathrm{H}_{2}$ with the accuracy resolution of $0.0165 \% \mathrm{H}_{2}$. Unfortunately, the best sensitivity found was about $0.1 \% \mathrm{H}_{2}$ that was presented in [7]. However, the special chirped FBG demonstrated the repeatability and a faster response time which was about $6 \mathrm{~s}$ when used as an advanced sensor. Consequently, our purpose in this research is to present a new robust hydrogen sensor design for disaster environment prevention in hydrogen leakage environments.

\section{Design of FBGs}

The most distinctive characteristic of fiber Bragg gratings is the flexibility offered for achieving desired spectral characteristics $[4,8]$. In the initial definition, the Bragg grating acted as a simple spectral filter that allowed a part of the incident signal to be reflected (shown in Fig. 1), which is expressed by [9]

$$
\lambda_{B}=2 n_{\text {eff }} \Lambda(z)
$$

where $\lambda_{B}$ is the central wavelength of the spectral band signal reflected by the Bragg grating, $n_{\text {eff }}$ is the 
effective refractive index in the unperturbed parts of the fiber Bragg grating length, and $\Lambda(z)$ is the grating period, which is naturally a constant when we talk about the uniform Bragg grating. In this paper, we consider three types of FBGs where the grating period is expressed as

$$
\begin{gathered}
\Lambda(z)=\Lambda_{0} \quad \text { conventional or uniform FBG } \\
\Lambda(z)=\Lambda_{0}\left(1+c_{p} z\right) \quad \text { chirped FBG } \\
\Lambda(z)=\left\{\begin{array}{l}
\Lambda_{0}\left(1-c_{p} z\right) \text { for }\left[0 \ldots \frac{z}{2}\right] \text { special chirped FBG } \\
\Lambda_{0}\left(1+c_{p} z\right) \text { for }\left[\frac{z}{2} \ldots z\right]
\end{array}\right.
\end{gathered}
$$

where $\Lambda_{0}$ is the nominal grating period, and $c_{p}(\mathrm{~nm} / \mathrm{cm})$ is the linear chirp coefficient of the grating period. And practically, the chirped FBG can be carried out by simple stretching movement in one direction $(+z)$, and the special chirped FBG can be carried out also by simple stretching movement but in the tow directions $( \pm z)$. In addition, we must assure the selection of design parameters for the confinement reasons, when the light waves propagate in the fiber core.

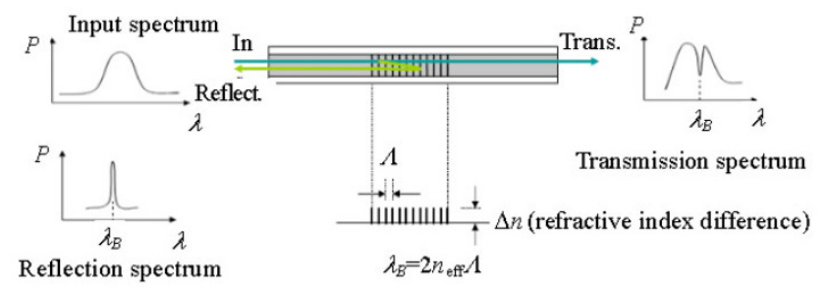

Fig. 1 Functional principle of FBG sensors.

In general, the Bragg gratings enable a transfer of the power between certain propagation modes inside the optical fiber. This is realized via the perturbation of the mode phase in a way to match with the phase of the other mode, which is in agreement with the phase condition [4]. When this is true, the reflected lights by each of Bragg grating levels interact in a constructive way in the contra-propagate direction. And so, several methods exist to describe the behavior of the Bragg grating in order to characterize them and to calculate their reflectivity. The most well-known is the coupled modes theory $[2,4]$. In our case, the fiber was mono-mode, and the interaction was recouped only between the contra-propagation modes. For the first design of the FBG, which was the uniform FBG, to make the reflectivity, we used the analytic solution. And for the chirped and special chirped FBGs, to make the reflectivity, we used the numerical and matrix solutions for the two coupled equations of the coupled modes theory [3]. Precisely, for the transfer matrix method solution, we used the division of the Bragg grating into uniform sections of the length $L_{u}$ which was verified by $L_{u}=L / N-1$ where $N$ is the number of total uniform sections, and $L$ is the total length of the grating. In addition, it must be that the length of each uniform section was much longer than $\Lambda_{0}$. Each uniform section was represented by a square matrix $B_{i}$ and by multiplying these entire matrixes, and a general matrix that described the full grating was obtained.

On the other hand, to make the chirped and special chirped FBGs work in the maximum efficiency, we assumed that each uniform Bragg section gave the maximum reflectance which is modulated as $[8,10]$

$$
R\left(\lambda, \Lambda_{z}\right)=\tanh ^{2}\left(K N_{p g} \Lambda_{z}\right)=\tanh ^{2}\left(K L_{u}\right)
$$

where $N_{p g}$ is the number of a periodical grating in the uniform section, $\Lambda_{z}$ is the grating period in the uniform section at the position $z$, and $k$ is the coupling coefficient (AC) which is expressed as $[2$, 4]

$$
K(z)=\frac{\pi \bar{\delta} n_{\mathrm{eff}}(z)}{\lambda_{B, z}} v
$$

where $\lambda_{B, z}$ is the central wavelength of each uniform Bragg section, $v$ is a classic value, and $\bar{\delta} n_{\text {eff }}(z)$ is the average index change in the refractive index, also for each uniform Bragg section. Subsequently, the term $\lambda_{B, z}$ can be expressed, based on the equation in [10], as

$$
\lambda_{B, z}=2\left(n_{\text {eff }}+\bar{\delta} n_{\text {eff }}(z)\right)\left[\Lambda_{0}-c_{p}\left(z-N_{T} \Lambda_{z} / 2\right)\right]
$$

with $L=N_{T} \Lambda_{z}, N_{T}$ is the total number of a periodical 
grating in the total grating length. And for the three types of FBGs, we used the following parameters: $\Lambda_{0}=0.53 \mu \mathrm{m}, c_{p}=1.5 \mathrm{~nm} / \mathrm{cm}, \bar{\delta} n_{\text {eff }}(z)=2.5 \times 10^{-4}$, $v=1, L=4 \mathrm{~mm}$, and $n_{\mathrm{eff}}=1.456$. From the simulation results of the reflective spectrum for the three shapes of FBGs, we had the same shape of the reflectivity but with different central wavelengths, which were very normal: $1.54336 \mu \mathrm{m}, 1.5465 \mu \mathrm{m}$, $1.54336 \mu \mathrm{m}$ for the uniform, chirped and special chirped FBGs, respectively. In addition, the factor, full width half maximum, (FWHM), which is a fundamental factor, was less than $0.5 \mathrm{~nm}$ for the uniform and chirped FBGs and was less than $0.25 \mathrm{~nm}$ for the special chirped FBG. This is very important, consequently, this narrow width allows increasing the multiplexing of several times with the same bandwidth.

In the next sections of this paper, the three shapes of FBGs will be discussed, which are used for the hydrogen leakage detection using the $\mathrm{Pd}$ (palladium) coating technique as the hydrogen sensitive film with the etched cladding. The hydrogen detection was realized via the mechanic effect, strain, exerted to the FBG length. So it is necessary to measure the strain sensitivity of each FBG shape. In addition, the measurement of the temperature sensitivity is also very important to compensate their effect and to control the sensor temperature for which it doesn't increase the temperature of the corrosion or explosion. These sensitivities lead to the shift in the Bragg wavelength, since the effective refractive index and the grating period are adjusted and deformed in the Bragg grating length, as indicated in the following expression $[9,11]$ :

$$
\frac{\Delta \lambda_{B}}{\lambda_{B}}=(\alpha+\xi) \Delta T+(1-\rho) \varepsilon
$$

where $\alpha$ is the fiber linear thermal or expansion coefficient (for silica, it is about $0.55 \times 10^{-6} /{ }^{\circ} \mathrm{C}$ ), $\xi$ is the thermo-optic coefficient (for silica, it is about $\left.8.6 \times 10^{-6} /{ }^{\circ} \mathrm{C}\right), \Delta T$ is the change in the temperature, $\varepsilon$ is the applied strain, and $\rho$ is the effective photo-elastic coefficient of the fibre core material. This last coefficient has a numerical value, which varies from 0.22 [9] to 0.26. Furthermore as we know, a good FBG sensor is that can give a linear response to the strain and/or temperature inside an acceptable range of the applied strains/temperature. And for our FBG sensors with the length of $4 \mathrm{~mm}$ and modulation index of $2.5 \times 10^{-4}$, we had absolutely a linear response to the strain and/or temperature where the central wavelength increased towards the infrared radiation in the linear way when the applied strain and/or temperature increased. And the sensitivities found were $1.39 \mathrm{pm} / \mu \varepsilon$ and $13.4 \mathrm{pm} /{ }^{\circ} \mathrm{C}, 2.24 \mathrm{pm} / \mu \varepsilon$ and $19 \mathrm{pm} /{ }^{\circ} \mathrm{C}, 1 \mathrm{pm} / \mu \varepsilon$ and $10 \mathrm{pm} /{ }^{\circ} \mathrm{C}$ for uniform FBG, chirped FBG and special chirped FBG, respectively. And from these obtained results, the thermal factor was dominated on the reflective spectrum which was absolutely normal. These values were very acceptable sensitivities in comparison with those of all publications.

\section{Design of hydrogen FBG sensors using $\mathrm{Pd} / \mathrm{Ti}$ coating technique}

In our case, we used slices of single-mode optical fibers of type SMF-28 of iXFiber society with reference IXC-SEN. The radius of the fiber core was equal to $4.6 \mu \mathrm{m}$, the radius of the optic cladding was in the order of $62.5 \mu \mathrm{m}$, and the radius of the mechanical protective cladding was $125 \mu \mathrm{m}$. Then, a Lambda Physik excimer laser (COMPex-150T) operating at $248 \mathrm{~nm}$ was used as the UV light source to write FBGs by the phase mask method, which were in a separate slice of the optical fiber. The uniform FBG was realized without modifications, the chirped FBG was realized by the simple stretching movement in one direction $(+z)$, and the special chirped FBG was realized also by the simple stretching movement but in the tow directions $( \pm z)$. Then, we had a uniform FBG with a central wavelength of $1.54336 \mu \mathrm{m}$ named FBG1, a chirped FBG with a central wavelength of $1.5465 \mu \mathrm{m}$ named FBG2, and a special chirped FBG 
with a central wavelength of $1.5424 \mu \mathrm{m}$ named FBG3. And we had noticed that the practical value of the central wavelength of the special chirped FBG was little different from the theoretical value $(1.54336 \mu \mathrm{m})$, which was due to the operation of the stretching movement in the tow directions $( \pm z)$ where a balance stretching movement in the tow directions was a difficult operation.

In this paper, we are interested in the technique of the etched cladding with a height of $62 \mu \mathrm{m}$, also the remainder of the cladding layer along each FBG with a height of $0.5 \mu \mathrm{m}$, and above this the Pd layer $(500 \mathrm{~nm})$ is realized as shown in Fig. 2. In this case, a special acrylic soft coating layer was used for the optical fiber to suppress the cladding mode. The remaining thickness of the cladding was controlled in each step by the coat thickness measurement device. Another solution of the coating presented in [5] which used the Pt-loaded $\mathrm{WO}_{3}$ (tungsten trioxide) coating technique where the preparation of $\mathrm{WO}_{3}$ was realized with the classic sol-gel method. We used the thick Pd layer to absorb hydrogen, inducing the strain change of the FBG1/2/3. To avoid the $\mathrm{Pd}$ coating layer from being peeled off when we had a high gas hydrogen concentration, we used the adhesive layer below the Pd layer in the three sensors. In this paper, this adhesive layer was titanium (Ti) layer with a low thickness in the order of $35 \mathrm{~nm}$ to strengthen the bond between the fiber cladding and $\mathrm{Pd}$ layer. Another solution was presented in [12] where we found the polyimide layer with the Ti layer as the adhesion layer. So our hydrogen FBG sensors consisted of the fiber core, fiber cladding $(0.5 \mu \mathrm{m})$, titanium (Ti) layer $(35 \mathrm{~nm})$ and Pd layer $(500 \mathrm{~nm})$. On the other hand, both of the Pd layer and $\mathrm{Ti}$ layer were coated by using magnetron sputtering where $\mathrm{Pd}$ and Ti grains were uniformly distributed. So we had high quality uniform metal layers in comparison with the other classic coating technologies like the sol-gel method, which protected the FBG and improved the sensitivity of the sensor.
Primarily, we tested FBG1, FBG2 and FBG3 sensors without any perturbation, and as a result, we had the same spectra as the spectra founded in Section 2. So the behavior of each FBG sensor remained the same using the $\mathrm{Pd}$ coating technique with the etched cladding if we had not a disruptive effect exerted on the FBG. In addition, in order to achieve the high sensitivity of each FBG sensor, the Pd layer should be as thick as possible, as revealed in [13]. However, this might cause a serious problem when FBG with the thick Pd layer was exposed to a high concentration of hydrogen where the $\mathrm{Pd}$ coating was peeled off because of the large internal stress exerted on the film due to volume expansion caused by the hydrogen absorption. In addition, the use of the Pd layer with a thickness of more than $700 \mathrm{~nm}$ decreased the efficiency of the adhesive layer which increased the peeled-off phenomenon, especially at the higher hydrogen concentration. Moreover, the use of the Pd layer with a thickness of lower than $200 \mathrm{~nm}$ decreased the desired influence on the spectral characteristics of the FBG. So the use of the Pd layer with the small thickness which was in the order of $500 \mathrm{~nm}$ was explained and required.

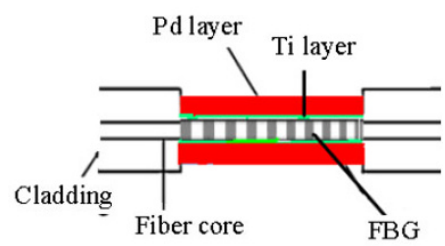

Fig. 2 Schematic diagram of the Pd/Ti-coated FBG hydrogen sensors with the etched cladding.

\section{Hydrogen detection FBG sensors}

\subsection{Definition}

Before using the sensors FBG1, FBG2 and FBG3, the three slices of the fiber containing these sensors were set in the $\mathrm{H}_{2}$ atmosphere for a week. Our experiment for examining our hydrogen sensors is shown in Fig. 3. The gas test cell was a Teflon tube, having two ports for the gas to flow in and out. The hydrogen gas flow rate through air was measured and controlled separately by the flow 


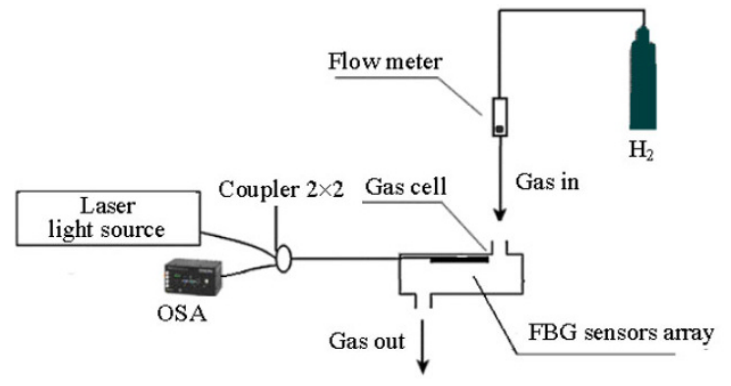

Fig. 3 Demonstration of the hydrogen sensing experiment with the FBG technology.

meter where the varying hydrogen concentrations were provided by changing the flowing rate of $\mathrm{H}_{2}$. In order to investigate the influence of different hydrogen concentrations on the shift in wavelengths of FBG1, FBG2 and FBG3, three FBG sensors arrays were inserted into the gas cell through a rubber cork and were fixed in a gas cell side, each one in a separate experiment. The first FBG sensors array contained the FBG1 hydrogen sensor and temperature compensation FBG sensor. The second contained the FBG2 hydrogen sensor and temperature compensation FBG sensor. And the third contained the FBG3 hydrogen sensor and also the temperature compensation FBG sensor. We used a temperature compensating FBG sensor, realized also by the phase mask method in each slice of the fiber containing the FBG hydrogen sensors, to eliminate the effect of the temperature when calculating the shift of each FBG hydrogen sensor. And as we have seen before, the central wavelengths of FBG1, FBG2 and FBG3 were $1.54336 \mu \mathrm{m}$, $1.5465 \mu \mathrm{m}$, and $1.5424 \mu \mathrm{m}$, respectively whereas the central wavelength of the temperature compensation sensor was set to $1.5413 \mu \mathrm{m}$. The choice of the temperature compensation sensor was related to the temperature sensitivity of each FBG hydrogen sensor, which had the same temperature sensitivity. On the other hand, each FBG sensors array was connected by a $3-\mathrm{dB}$ coupler to a laser light source and an optical spectrum analyzer (OSA) SM-130, manufactured by Micron Optics, whose operating range was from $1510 \mathrm{~nm}$ to $1590 \mathrm{~nm}$ with a resolution of $1 \mathrm{pm}$. All experiments were performed at the room temperature of $25^{\circ} \mathrm{C}$ and atmospheric pressure.

\subsection{Discussion}

When Pd reacts with hydrogen, its optical absorption and refractive index will change. Since the physical stress caused by the volume change of Pd has little effect on the sensor's performances via the mechanic effect, so shifts in the wavelength are realized. These shifts in wavelengths of FBG1, FBG2 and FBG3 were observed with different hydrogen concentrations: $0.1 \%-4 \%$. Figure 4

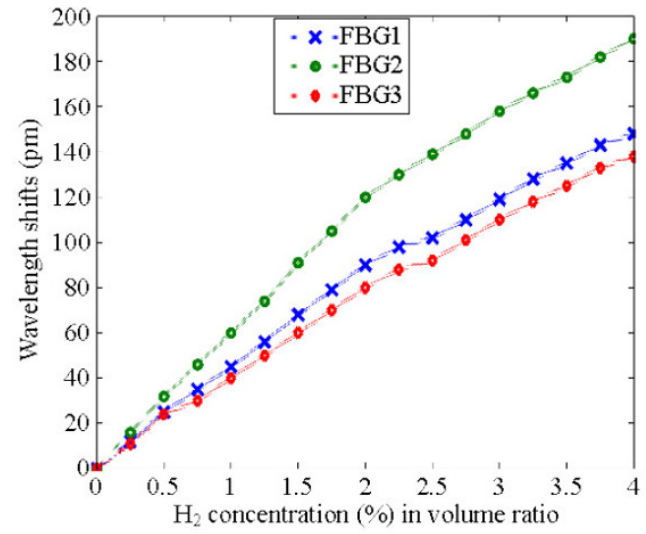

Fig. 4 FBG1, FBG2 and FBG3 wavelength shifts response to hydrogen concentrations.

indicates these shifts response after eliminating the temperature effect via the temperature compensating FBG sensor. Each FBG we used had the temperature sensitivity in the order of $13.4 \mathrm{pm} /{ }^{\circ} \mathrm{C}, 19 \mathrm{pm} /{ }^{\circ} \mathrm{C}$ and $10 \mathrm{pm} /{ }^{\circ} \mathrm{C}$ at the room temperature. From the curves, the wavelengths of FBG1, FBG2 and FBG3 increased almost linearly as a function of hydrogen concentrations in the range of $0.1 \% \mathrm{H}_{2}-4 \% \mathrm{H}_{2}$. The added titanium (Ti) layer did not affect the sensors sensitivity. In addition, experimental results showed the highest sensitivity existing with the chirped FBG, FBG2, rather than the uniform FBG, FBG1 and the special chirped FBG, FBG3. This is explained as FBG2 had the greater strain sensitivity in comparison with those of the two other FBGs, FBG1 and FBG3, where the changes in the grating period and refractive index were more important. 
Especially in the range of $0.1 \% \mathrm{H}_{2}-2 \% \mathrm{H}_{2}$, the sensitivities were $45 \mathrm{pm} / 1 \% \mathrm{H}_{2}, 60 \mathrm{pm} / 1 \% \mathrm{H}_{2}$ and $40 \mathrm{pm} / 1 \% \mathrm{H}_{2}$ of FBG1, FBG2 and FBG3, respectively. In addition, in this range, the response time of these hydrogen sensors was $11 \mathrm{~s}, 9 \mathrm{~s}$ and $4 \mathrm{~s}$ of FBG1, FBG2 and FBG3, respectively. The response time was calculated from the hydrogen flowing into the gas room until each FBG reaching the maximum wavelength. Afterwards, the sensitivity and the response time decreased in the range of $2 \% \mathrm{H}_{2}-4 \% \mathrm{H}_{2}$, which were $30 \mathrm{pm} / 1 \% \mathrm{H}_{2}$ and $13 \mathrm{~s}, 40 \mathrm{pm} / 1 \% \mathrm{H}_{2}$ and $10 \mathrm{~s}$ and $30 \mathrm{pm} / 1 \% \mathrm{H}_{2}$ and $8 \mathrm{~s}$ for FBG1, FBG2 and FBG3, respectively. And considering the resolution of our OSA which was $1 \mathrm{pm}, 0.022 \% \mathrm{H}_{2}$ could be detected in air by the FBG1, $0.0165 \% \mathrm{H}_{2}$ could be detected in air by the FBG2, and $0.025 \% \mathrm{H}_{2}$ could be detected in air by the FBG3 in the range of $0.1 \% \mathrm{H}_{2}-2 \% \mathrm{H}_{2}$. It has been demonstrated also that the wavelength shift of FBG1/FBG2/FBG3 was more than 146/190/137nm when the hydrogen concentration was $4 \%$ in the volume ratio at the room temperature. So it was reported from the experiment results that the chirped FBG hydrogen sensor coated with $\mathrm{Pd} / \mathrm{Ti}$ with the etched cladding presented the highest sensitivity with the linear response, however the special chirped FBG hydrogen sensor coated with $\mathrm{Pd} / \mathrm{Ti}$ with the etched cladding presented the faster response time with the linear response when the hydrogen concentration was below the explosive limit $\left(4 \% \mathrm{H}_{2}\right.$ in the volume ratio). In addition, all the sensitivities founded in this paper were more efficient in comparison with those published with the uniform FBG sensor coated with the Pd layer without the etched cladding. This was due to the structures of our FBG sensors with the etched cladding.

On the other hand, each FBG hydrogen sensor can be a combustion source when the hydrogen concentration is more than $4 \% \mathrm{H}_{2}$ where the temperature around each FBG increases more than $100{ }^{\circ} \mathrm{C}$. So to obtain the FBG hydrogen sensor with the intrinsic safety, the temperature increasing with $\mathrm{H}_{2}$ around each FBG should be controlled at a proper value. Subsequently, the presence of the temperature compensation FBG sensor is very important to compensate the temperature effect and to control the sensor temperature so as not to increase the temperature of corrosion or the explosion. Moreover, in some cases, the hydrogen concentration may be more than $4 \%$ in the volume percentage, so the mentioned experiments are not suitable for applications in these fields. Figure 5

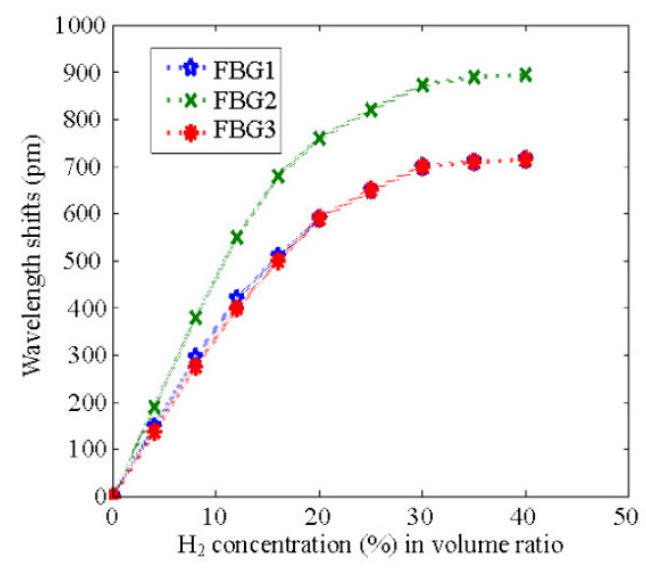

Fig. 5 FBG1, FBG2 and FBG3 wavelength shifts response to high hydrogen concentrations.

illustrates the responses of FBG1, FBG2 and FBG3 under the higher hydrogen concentration by simulation where the data acquisition was made by the LabVIEW program. And from this figure, when the hydrogen concentration increased, each FBG shifted to the longer wavelength, but the wavelength shift increased slowly at the high hydrogen concentration with a nonlinear response. Owing to the structures of FBG1, FBG2 and FBG3, when the hydrogen increased to $30 \%$, the Pd had been almost totally consumed, and the central wavelength was fixed. Despite this result, the FBG2 and FBG3 sensors were efficiency even under the almost pure hydrogen atmosphere. The first FBG, FBG2, gave a more hydrogen sensitivity, and the second FBG, FBG3, gave a faster response time. So they could be used to monitor the hydrogen leakage at all hydrogen concentrations. But it is more suitable to 
measure low hydrogen concentrations in air. And the fastest response time of FBG3 obliged us to enhance the strain sensibility; this is the advanced hydrogen FBG sensor which will be presented in the next section of this paper.

\section{Advanced hydrogen detection FBG sensors}

An advanced hydrogen FBG sensor was developed based on the FBG3. The shape of this sensor is presented in Fig. 6 where we had the shape of FBG3 with 2 layers of $\mathrm{Pd} / \mathrm{Ti}$ on the two sides of the FBG3 with a length of $4 \mathrm{~mm}$ each. In this way, we increased the strain sensibility, mechanic effect, so the hydrogen sensitivity. Figure 7 illustrates the

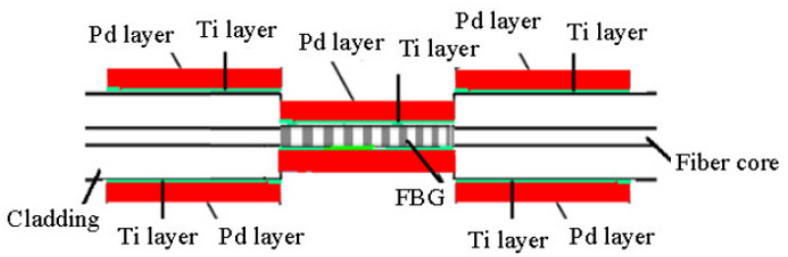

Fig. 6 Shape of the advanced FBG: FBG3 in the middle of the length of $4 \mathrm{~mm}$ and 2 layers of Pd/Ti with the length of $4 \mathrm{~mm}$ each on the two sides.

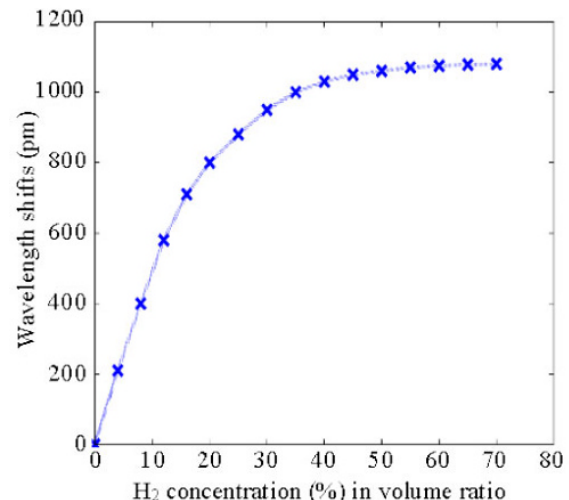

Fig. 7 Advanced FBG wavelength shifts response under low and high hydrogen concentrations.

response of this advanced FBG under low and high hydrogen concentrations by simulation. Also, as the previous section, the data acquisition was made by the LabVIEW program. From the curve, we had a sensitivity of $60 \mathrm{pm} / 1 \% \mathrm{H}_{2}$ in the range of $0.1 \% \mathrm{H}_{2}-$ $4 \% \mathrm{H}_{2}$ which was better in comparison with those presented in the previous sections of this paper with the faster response time which was in the order of $6 \mathrm{~s}$. In addition, the global sensitivity increased significantly where we had a shift over $1 \mathrm{~nm}$ when we had $40 \%$ of $\mathrm{H}_{2}$ in the volume ratio, and this is very important to detect easily any hydrogen leakage.

Our sensor is used for the disaster environment prevention by locating the leakage position of hydrogen more quickly and accurately than the conventional sensors, which is very important and essential to save people especially in environmentally sensitive positions and in the environment where the hydrogen presents as the nuclear centers, in aerospace vehicles, hydrogen dissolved in transformer gas and in petroleum oils. As an example of the real application, NASA Langley has used 20 palladium tubes coated with FBGs on the board of the Space Shuttle Discovery on STS-96 [14, 15] for hydrogen sensing, see Fig. 8. With this fiber optic network, they could quantitatively detect hydrogen leaks in the aft bay of the orbiter during the hydrogen tanking and at the moment of the vehicle operation. But the most important issue is the response time which must be within an acceptable level to avoid any tragedy to happen. At present, the fastest response FBG hydrogen sensor is based on the Pt-loaded $\mathrm{WO}_{3}$ coating [5] undergoing an exothermic reaction in the hydrogen atmosphere: $10 \mathrm{~s}$, and the most of FBG hydrogen sensors published are based on the volume expansion of $\mathrm{Pd}$ or their composite films have a response time more than $30 \mathrm{~s}$. The main reason for the long response time is due to the slow hydrogen diffusion rate of the hydrogen sensitive film. Moreover, we have demonstrated experimentally in this paper that our advanced FBG hydrogen sensor using the etched cladding with the Pd coating as a hydrogen sensitive film gave also a fast response time compared to those of Pt-loaded $\mathrm{WO}_{3}$ with the high and reliable sensitivity especially in low hydrogen concentrations. 


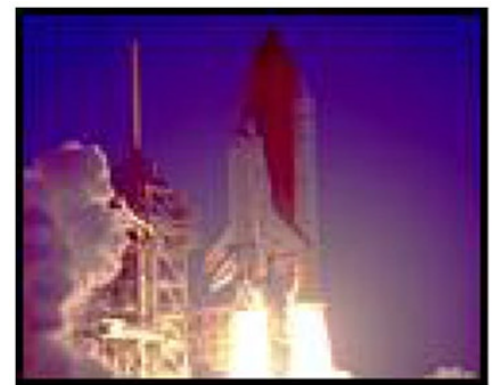

Fig. 8 Space Shuttle Discovery on STS-96 using 20 gratings coated with Palladium tubes for hydrogen sensing [15].

\section{Conclusions}

New robust FBG hydrogen sensors with the length of $4 \mathrm{~mm}$ based on the Pd material $(500 \mathrm{~nm})$ as the hydrogen sensitive film and the Ti layer $(35 \mathrm{~nm})$ as the adhesive layer were developed, and their sensing characteristics have been investigated. And from experimental results, with the etched cladding technique, better results were obtained especially in the low concentration range of hydrogen with a resolution reaching $60 \mathrm{pm} / 1 \% \mathrm{H}_{2}$. With the FBG demodulator resolution of $1 \mathrm{pm}$, the high precision to reduce the hydrogen concentration variation could be detected: $0.0165 \% \mathrm{H}_{2}$. Also it was demonstrated that these sensors were safe even under the higher hydrogen concentration. These sensors are very promising for the disaster environment prevention by locating the leakage position of hydrogen more quickly and accurately which is very important to save people. Especially with the advanced sensor, we had a response time reaching $6 \mathrm{~s}$ which is the better response time found up to now. And with dense wavelength division multiplxing (DWDM) technique, all the bodies are recovered against the hydrogen leakage with several FBG hydrogen sensors like in aerospace vehicles: it is a guarantee. On the other hand, it should be noted that an increase in the wavelength when the hydrogen concentration increases is less efficient in the repeated measurement. This is due to the presence of the blistering in the Pd layer and the presence of hydrogen molecules from the previous measurement where the central wavelength of the FBG sensor does not return to the center previously defined without any outside perturbation with an increase in this return time when it is exposed in air for recovery. This less efficiency in the repeated measurement increases from the measurement to the next measurement. However, despite this drawback, the advanced sensor still has the potential for monitoring hydrogen leaks several successive times due to its high precision and quick response time before change it if any dangerous value is detected and if the Pd layer is totally peeled off.

\section{Acknowledgment}

This work was supported by the laboratory of Advanced Materials and Quantum Phenomena, Faculty of Sciences of Tunis, Tunis-EL Manar University in 2012-2013 funded by the Ministry of Higher Education and Scientific Research, Tunisia.

Open Access This article is distributed under the terms of the Creative Commons Attribution License which permits any use, distribution, and reproduction in any medium, provided the original author(s) and source are credited.

\section{References}

[1] Y. Tana ka and T. Kurokawa, "Disaster prevention by photonics - toward realization of safe and secure society," presented at the EOSAM 2012, Aberdeen, Scotland, UK, Sep. 25-28, 2012.

[2] K. O. Hill and G. Meltz, "Fiber Bragg grating technology fundamentals and overview," Journal of lightwave Technology, vol. 15, no. 8, pp. 1263-1276, 1997.

[3] C. Lu, J. Cui, and Y. Cui, "Reflection spectra of fiber Bragg grating with random fluctuation," Optical Fiber Technology, vol. 14, no. 2, pp. 97-101, 2008.

[4] M. Suleiman, "Design of an optoelectronic sensor by optical interferometry retro injection for the demodulation of the signals in optical fiber Bragg gratings, " M.S. thesis, National Polytechnic Institute of Toulouse, Toulouse University, France, 2008.

[5] M. Yang, Z. Yang, J. Dai, and D. Zhang, "Fiber optic hydrogen sensor with sol-gel $\mathrm{WO}_{3}$ coatings," Sensors and Actuators B: Chemical, vol. 166-167, pp. 
632-636, 2012.

[6] B. Sutapun, M. Tabib-Azar, and A. Kazemi, "Pd-coated elastooptic fiber optic Bragg grating sensors for multiplexed hydrogen sensing," Sensors and Actuators B: Chemical, vol. 60, no. 1, pp. 27-34, 1999.

[7] S. F. Silva, L. Coalho, O. Frazao, J. L. Santos, and F. X. Malcato, "A review of palladium based fiber-optic sensors for molecular hydrogen detection," IEEE Sensors Journal, vol. 12, no. 1, pp. 93-102, 2012.

[8] H. S. Phing, J. Ali, R. A. Rahman, and B. A. Tahir, "Fiber Bragg grating modeling, simulation and characteristics with different grating lengths," Malaysian Journal of Fundamental and Applied Sciences, vol. 3, no. 2, pp. 167-175, 2007.

[9] A. D. Kersey, M. A. Davis, H. J. Patrick, M. LeBlanc, K. P. Koo, C. G. Askins, et al., "Fiber grating sensors," Journal of Lightwave Technology, vol. 15, no. 8, pp. 1442-1463, 1997.

[10] O. Wi, G. Farrell, and Y. Semenova, "Simple design technique for a triangular FBG filter based on a linearly chirped grating," Optics Communications, vol. 283 , no. 6, pp. 985-992, 2010.

[11] R. Suresh and S. C. Tjin, "Effect of dimensional and material parameters and cross-coupling on FBG based shear force sensor," Sensors and Actuators A: Physical, vol. 120, no. 1, pp. 26-36, 2005.

[12] G. M. Ma, C. R. Li, Y. T. Luo, R. D. Mu, and L. wang, "High sensitive and reliable fiber Bragg grating hydrogen sensor for fault detection of power transformer," Sensors and Actuators B: Chemical, vol. 169, no. 5, pp. 195-198, 2012.

[13] B. Michael, P. C. Kevin, B. Matrika, R. S. Philip, and M. Mokhtar, "Active fiber Bragg grating hydrogen sensors for all-temperature operation," IEEE Photonics Technology Letters, vol. 19, no. 5, pp. 255-259, 2007.

[14] J. P. Moore, B. A. Childers, M. E. Froggatt, A. L. Cook, N. C Coffey, L. J. Coen, et al., "An overview of the fiber optic sensing system for hydrogen leak detection in the space shuttle discovery on STS-96," presented at OSA Proceedings Bragg Gratings, Photensensitivity and Poling in Glass Waveguides, Stuart FL, Sep. 23-25, 1999.

[15] M. Yu, "Fiber optic sensor technology," presented at 26th International Modal Analysis Conference (IMAC XXVI), University of Maryland, Orlando, Florida, USA, Feb. 6, 2008. 